Koloman Brenner*

ELTE Budapest
UDK 323.15(439=112.2):37

DOI: 10.4312/linguistica.60.2.105-116

\title{
NEUE TENDENZEN IM BILDUNGSSYSTEM DER DEUTSCHEN MINDERHEIT IN UNGARN
}

\section{EINLEITUNG}

Die deutsche Minderheit in Ungarn gehört zu den relativ großen deutschen Gemeinschaften in Ost-Mittel-Europa, die eine lange historische Entwicklung vorweisen kann (vgl. Brenner 2018 und Scheuringer 2015). Im heutigen Ungarn gibt es drei größere Siedlungsgebiete, wo Angehörige der deutschen Minderheit in höherer Anzahl leben: Westungarn entlang der österreichischen Grenze, das Ungarische Mittelgebirge (vom Ofner Bergland bis zum Plattensee-Oberland) mit den Zentren Ofen/Buda und Zirc bzw. Südungarn mit dem Zentrum Fünfkirchen/Pécs. Die heutige Lage der deutschen Minderheit in Ungarn widerspiegelt das Ergebnis von langwierigen sprachlichen, kultur- und minderheitenpolitischen bzw. gesellschaftlichen Veränderungen. Die Tatsache, dass ab dem 16. Jahrhundert bis 1918 das ungarische Königreich in unterschiedlichen juristischen Konstruktionen mit dem Habsburger-Reich verbunden war, brachte ebenfalls in mannigfaltiger Weise einen sprachlichen und kulturellen deutschen Einfluss mit sich. In diesem Prozess entstanden die deutschen Sprachinseln in Ungarn. Nach 1945 wurden die intensiven deutsch-ungarischen sprachlichen und kulturellen Kontakte gewaltsam unterbrochen, die Vertreibung von etwa 200.000 Deutschen anschließend verursachte ein zusätzliches Trauma. ${ }^{1}$

Im folgenden halben Jahrhundert können wir zwei Entwicklungsphasen auseinanderhalten: Erstens die sog. „,schweren Jahrzehnte“, die 1950er, 1960er und 1970er Jahre, zweitens etwa seit Mitte der 80er Jahre des 20. Jahrhunderts die neue Phase einer eher positiven Entwicklung (vgl. Knipf/Erb 1998). Über den Bildungsbereich dieser Jahrzehnte sind bis jetzt nur spärliche Untersuchungen in der Fachliteratur zu finden (vgl. Tóth 2015). Es ist aber wichtig zu erwähnen, dass in den ersten Jahren der „schweren Jahrzehnte" Deutsch aus den Schulen und Kindergärten verbannt wurde. Erst in der zweiten Phase beginnt ein langsamer und nicht eindeutig erfolgreicher Prozess Richtung bilingualer Schulen, auch auf der Mittelschulebene. Sogar im Kindergartenbereich gibt es erste Schritte in Richtung einer zweisprachigen Erziehung (vgl. Brenner 1994). Wenn wir also die sprachliche Situation der deutschen Minderheit in Ungarn heutzutage generell beobachten, ergibt sich folgendes Bild: Die deutschen Dialekte sind im Rückzug, der Sprachverlust und die Assimilation ist aber in den drei angeführten Siedlungsgebieten z.

brenner.koloman@btk.elte.hu

$1 \mathrm{Zu}$ der ausführlichen Geschichte der Deutschen in Ungarn siehe das neue Standardwerk in zwei Bänden von Seewann (2012). 
T. unterschiedlich vorangeschritten. Die Dialektkompetenz ist eindeutig abhängig vom Alter, die anderen sozialen Faktoren modifizieren lediglich das Gesamtbild. Von der ältesten Generation angefangen registrieren wir eine graduelle Einengung der Kompetenz, die produktive Verwendung wird in den anderen Generationen immer geringer, bei der jungen Generation beschränkt es sich fast nur auf ritualisierte Sprechsituationen (vgl. Knipf/Erb 1998). Die Einengung der dialektalen deutschen Kompetenz ist in Westungarn nicht so vorangeschritten wie in der Umgebung von Budapest, allerdings im Vergleich zu Südostungarn, wo auch in der mittleren Generation breite Schichten der Ungarndeutschen produktiv und rezeptiv die deutsche Dialektform beherrschen und sogar in der jüngeren Generation nicht nur vereinzelt diese Kompetenz erscheint, ist der Prozess stärker ausgeprägt (vgl. Brenner 2003 und 2016).

Der vorliegende Aufsatz will die neuen Tendenzen im Bildungsbereich und ihre Rolle im Revitalisierungsprozess der deutschen Sprache und Kultur der betroffenen nationalen Minderheit in Ungarn darstellen und analysieren. Dies ist auch deswegen eine relevante Fragestellung, weil im Vergleich mit anderen deutschen Minderheiten in Ost-Mittel-Europa, der deutsche Fremdsprachenunterricht und auch der Unterricht von Deutsch als Minderheitensprache eine spezifische Entwicklung vorweisen kann. In Schlesien (Polen) auf der einen Seite, wo Angehörige der deutschen Minderheit in relativ hoher Anzahl und in einer kompakten Siedlungsstruktur leben, wurde der Unterricht der deutschen Sprache bis zu der politischen Wende nicht möglich gemacht. Auf der anderen Seite war im kommunistischen Rumänien der muttersprachlich-deutsche Bildungsweg trotz aller ideologischen Hindernisse und Beeinflussungen aufrechterhalten worden. Ungarn zeichnete sich durch einen langsamen Ausbau des deutschen Minderheitenunterrichts ab den 60er und intensiver ab den 70er Jahren des 20. Jahrhunderts. Allerdings geschah dies nicht flächendeckend und planmäßig, so dass sich der Deutschunterricht auf nur einige Wochenstunden beschränkte. Des Weiteren verschwommen die Grenzen zwischen DaF-Unterricht und DaM-Unterricht, was zu zusätzlichen spezifischen Problemen und Entwicklungen führte. Nicht zuletzt sind in den letzten Jahren eindeutig positive Tendenzen im ungarndeutschen Bildungswesen zu beobachten, was die Übernahme von Kindergarten- und Grundschulinstitutionen durch die örtlichen deutschen Selbstverwaltungen anbelangt. Die oben angeführten spezifischen Tendenzen werden im vorliegenden Aufsatz geklärt und gedeutet.

\section{MINDERHEITEN- UND BILDUNGSPOLITISCHE RAHMENBEDINGUNGEN}

Um die aktuellen Tendenzen im Minderheiten-Bildungswesen der Deutschen in Ungarn richtig zu deuten und bewerten zu können, müssen zuerst die allgemeinen minderheiten- und bildungspolitischen Rahmenbedingungen und ihre Entwicklung seit der Zeit der politischen Wende in Ost-Mittel-Europa geschildert werden. In den letzten fast 30 Jahren veränderten sich die Möglichkeiten der (minderheiten)politischen Interessenvertretung der Ungarndeutschen. Auf diesem Gebiet geschah auch dieser Übergang 
in Ungarn etwas fließender - im Vergleich zu anderen früheren Ostblockstaaten. Auch im politischen Bereich sind die Signale in den 1980er Jahren nicht zu verkennen: Nachdem die Bundesrepublik Deutschland als wichtiger Handelspartner eine immer größere strategische Bedeutung für Ungarn bekommen hat, mündet diese Entwicklung darin, dass im Oktober 1985 mit kräftiger Unterstützung des damaligen deutschen Außenministers Hans-Dietrich Genscher der erste offiziell gegründete deutsche Minderheitenverein in Osteuropa ins Leben gerufen wurde: der Kulturverein Nikolaus Lenau e.V. in Fünfkirchen/Pécs.

Die erste frei gewählte ungarische Regierung unter Ministerpräsident Antall schickte sich 1990 an, die bedeutenden ungarischen Minderheitengemeinschaften in den Nachbarstaaten kräftig zu unterstützen. Um diese Zielsetzung international besser begründen zu können, wurde ein „beispielhaftes Minderheitengesetz“ ins Visier genommen, innen- und außenpolitische Entwicklungen prägten das Bild ebenfalls. Die Verabschiedung des Minderheitengesetzes im Jahre 1993 und die darauffolgende neue Struktur der sog. Minderheitenselbstverwaltungen (Körperschaften, die die kulturelle Autonomie durch Wahlen verwirklichen, z. B. Schulträgerinnen werden können) führten zu einem Neubeleben der Tätigkeiten der Volksgruppen in allen Lebensbereichen. Bei den Kommunalwahlen im Herbst 1994 sind also die ersten Wahlen der örtlichen Minderheitenselbstverwaltungen durchgeführt worden. Im März 1995 wählten im nächsten Schritt die sog. Elektoren der deutschen Minderheit das erste Mal die Vollversammlungsmitglieder der Landesselbstverwaltung der Ungarndeutschen (LdU). Sie ist seitdem das höchste kulturelle und politische Repräsentationsorgan der deutschen Minderheit in Ungarn.

Die Kandidaten der Minderheitenwahlen stellten in der Regel die örtlichen oder landesweiten Kulturvereine der Deutschen, sodass es zu einer gewissen Verdoppelung der früheren Strukturen kam, da dieselben Personen in den Gremien wirkten. Allerdings kann die Entwicklung auch so bewertet werden, dass die zivile Gesellschaft bei den Kommunalwahlen die Minderheitenpolitiker gewählt hat, die ihre Interessen auf der politischen Ebene vertreten, da die Minderheitenselbstverwaltungen von ihrer juristischen Position gesehen als öffentlich-rechtliche Körperschaften wesentlich mehr Kompetenzen als Vereine haben. Die Kompetenzen dieser neuen Gremien wurden nur nach und nach ausgelotet. Die Existenz der deutschen Minderheitenselbstverwaltungen sicherte eine zwar bescheidene, aber staatlich definierte Grundfinanzierung, die vor allem in kleineren Gemeinden durch Unterstützung der in den einzelnen Ortschaften gewählten ungarndeutschen Vertreter funktionierenden ungarndeutschen Tanzgruppen, Chöre, Blaskapellen usw. eine positive Wirkung und eine gewisse Planungssicherheit im Minderheitenkulturleben mit sich brachte. Durch die zusätzlichen Kompetenzen der Minderheitenselbstverwaltungen und durch den „Wahlkampf“ für die Minderheitenwahlen präsentierten sich auch für die breitere Öffentlichkeit in den verschiedenen betroffenen Ortschaften Vertreter der Deutschen in einer neuen, gestärkten Position, wenn auch in den ersten Jahren die örtlichen Administrationen immer wieder Probleme hatten, die Kompetenzen der Minderheitenselbstverwaltungen anzuerkennen und ihren Pflichten nachzukommen (vgl. auch Demeter Zyzon 1999 bzw. Dobos (2009). 
Das Mitbestimmungsrecht (de facto Vetorecht) der Minderheitenselbstverwaltungen bei der Einstellung der Schul- und Kindergartendirektoren verursachte z. B. in der Stadt Ödenburg im Jahre 2002 sogar Konflikte auf der höchsten politischen Ebene, als ein der deutschen Sprache nicht mächtiger Schuldirektor als Leiter der deutschen Nationalitätenschule vom Stadtrat gewählt wurde. In den Minderheitenbildungsinstitutionen hatten nämlich laut der Regelungen des Minderheitengesetzes die Minderheitenselbstverwaltungen auch hinsichtlich der Annahme des pädagogischen Programms ein Mitbestimmungsrecht, auch wenn davon die Gremien nicht zu häufig Gebrauch machten. Dieses Recht wurde infolge der neuen Gesetzgebung nach 2010 den Gremien entzogen, insofern wurde der Einfluss der Körperschaften auf die pädagogischen Leistungen eingeschränkt. Zur Situation und Analyse des Bildungssystems der Ungarndeutschen nach der Wende siehe auch Brenner (1994 und 2003); Müller (2011 und 2012) bzw. Takács (2013).

\section{AKTUELLE TENDENZEN IM DEUTSCHEN MINDERHEITEN- BILDUNGSWESEN IN UNGARN}

Die heutige Lage der schulischen Einrichtungen der deutschen Minderheit in Ungarn spiegelt das Ergebnis von langwierigen kultur- und minderheitenpolitischen bzw. gesellschaftlichen Veränderungen wider. In den Institutionen, wo auch Angehörige der deutschen Minderheit einen gesteuerten Weg des Lernens erreichen können, herrscht nach wie vor ein recht unterschiedliches Bild, was die Qualität der Erziehung und des Unterrichts anbelangt. Wie oben angeführt, entwickelte sich das Schulwesen der deutschen Minderheit in Ungarn seit den 80er Jahren der 20. Jahrhunderts zwar unter besseren Rahmenbedingungen, aber es fehlten die klaren gesetzlichen und fachlichen Fundierungen des Minderheitenunterrichts. In den 90er Jahren des 20. Jahrhunderts herrschte z. T. eine chaotische Situation sogar im Bereich der Terminologie. Begriffe wie Nationalitätenunterricht, Minderheitenunterricht, Sprachunterricht, zweisprachiger Unterricht usw. wurden sowohl beim Unterrichtsministerium als auch bei den betroffenen Institutionen bzw. bei den Gemeinde- und Stadträten, die als Institutionsträgerinnen funktionieren, unterschiedlich verwendet und ausgelegt.

Hinter den anmutenden statistischen Zahlen des Unterrichtsministeriums über die Anzahl der Schüler, die an einem deutschen Minderheitenunterricht teilnehmen, steckte eine kunterbunte Realität, wobei die meisten Kinder von Angehörigen der deutschen Minderheit keine Schule oder keinen Kindergarten besuchen, die ihren spezifischen Ansprüchen entsprechen würden. Der typische Fall v. a. in kleineren Ortschaften - und bekanntlich leben die meisten Ungarndeutschen in solchen Ortschaften - war, dass in der Grundschule (die sich stolz Nationalitätenschule nannte) de facto Deutsch als Fremdsprache unterrichtet wurde. Vielerorts ist dies auch nur in einem Klassenzug der Fall und alle anderen Stunden bzw. die außerschulischen Aktivitäten liefen in ungarischer Sprache.

Um die Ausgangssituation ein wenig detaillierter zu schildern, sollten die Zahlen aus einer Umfrage des Kultusministeriums, die in 209 Schulen mit einem 
Minderheitenunterricht (entweder Sprachunterricht oder zweisprachiger Unterricht) im Jahre 1992 durchgeführt wurde, zitiert werden: In 10 befragten Schulen fand in 6 Wochenstunden ein Deutschunterricht statt, in 3 Schulen waren es 5 Wochenstunden, in 5 Schulen 4 Wochenstunden und in nur zwei Schulen drei Stunden pro Woche. In den restlichen Schulen gab es dementsprechend in einer oder zwei Wochenstunden Deutschunterricht (vgl. Brenner 1994: 138).

Das ungarische Minderheitengesetz aus dem Jahre 1993, das 2005 modifiziert wurde, bot den Minderheitengemeinschaften in Ungarn prinzipiell die Rahmenbedingungen einer sog. kulturellen Autonomie. In den untersuchten Jahrzehnten haben die sog. Minderheitenselbstverwaltungen zwar viele positive Impulse in den Kreisen der Ungarndeutschen bewirkt, aber im Unterrichtswesen sind ab Ende der 90er Jahre des 20. Jahrhunderts neue, eher negative Tendenzen zu verzeichnen (vgl. auch Demeter Zyzon 1999 und Molnár 1998). Hinzu kommt außerdem, dass der normale Prozess der Weitergabe der Sprache in den Familien der Angehörigen der deutschen Minderheit kaum mehr möglich ist. Aus den angeführten Gründen sind zwei bzw. drei Generationen aufgewachsen, die dadurch gekennzeichnet sind, dass prozentual gesehen relativ wenige eine deutsche sprachliche Varietät authentisch beherrschen.

Zweisprachige Klassenzüge wurden aufgelöst, der allgemeine Rückgang der Kinderzahlen führte in den früheren Jahren zur Schließung von Schulen. Der notorische Mangel an gut ausgebildeten Deutschlehrern, das vollkommene Fehlen einer deutschsprachigen Ausbildung für Fachlehrer, die andauernde Problematik der entsprechenden Lehrwerke im Minderheitenunterricht usw. sind Signale dieser negativen Richtung. Die Landesselbstverwaltung der Ungarndeutschen (LdU) registrierte diese negativen Tendenzen und beschloss 1998, dass als Verwirklichung der kulturellen Autonomie die LdU als Trägerin von wichtigen schulischen und kulturellen Institutionen funktionieren sollte. Im ersten Jahrzehnt des 21. Jahrhunderts wurden wichtige Schulzentren in eigene Trägerschaft der deutschen Minderheit übernommen. Seit 2004 funktioniert in dieser Form das Valeria-Koch-Bildungszentrum in Fünfkirchen/Pécs (Südungarn) und das Friedrich-Schiller-Gymnasium, Berufliches Gymnasium und Schülerwohnheim in Werischwar/Pilisvörösvár bei Budapest. Als Bahnbrecher ist das Fünfkirchner Zentrum zu bezeichnen, das seit einigen Jahren konsequent einsprachig deutsche Kindergartengruppen startete. Im Jahre 2017 fing die einsprachig deutsche Ausbildung demnach schon in der fünften Klasse der Grundschule an, und dieser Zweig der Minderheitenausbildung ist der Garant einer erfolgreichen Neubelebung der Sprache und Kultur der Ungarndeutschen.

Seit September 2015 wird das Deutsche Nationalitätengymnasium Budapest ebenfalls von der LdU getragen. Das Ungarndeutsche Bildungszentrum (UBZ) in der Stadt Baja wird von einer Stiftung unterhalten, hier nimmt die LdU ebenfalls an der gemeinsamen Trägerschaft teil. Eine interessante neue Tendenz bezüglich der Zusammenarbeit der deutschen Firmen in Ungarn mit den ,alteingesessenen“ Angehörigen der deutschen Minderheit repräsentiert die in Raab gegründete Audi Schule. Seit September 2014 ist die Schulträgerin die Audi Hungaria Schule Öffentliche Träger- und Betreiberstiftung, gegründet von der LdU und in Mitträgerschaft der Audi Hungaria AG. 
Die neuste und positivste Tendenz im Bildungsbereich der Deutschen in Ungarn begann mit einer eher allgemein als negativ eingestuften Veränderung. Die seit 1990 kommunal getragenen Grundschulen und Kindergärten bekamen im Jahre 2013 eine neue, zentralstaatliche Trägerschaft: Ab diesem Schuljahr wurden also dieselben nicht mehr von den einzelnen Gemeinde- oder Stadträten getragen, sondern diese Funktion übernahm die sog. „Klebelsberg Zentrale für Institutionhaltung“ (ihre ungarische Abkürzung heißt KLIK), wie die deutsche Bezeichnung in der Gründungsurkunde etwas unglücklich formuliert heißt. In den kleinen Ortschaften konnten die Gemeinde- und Stadträte nach diesen Veränderungen nicht wie gewohnt die pädagogische Arbeit und die Berufung der Institutionsleiter beeinflussen. Des Weiteren entstand innerhalb von der KlebelsbergZentrale ein langer Dienstweg, bis Alltagsprobleme behoben werden konnten. Dies verursachte landesweit eine gewisse Unzufriedenheit mit diesem zentralisierten System der Schulträgerschaften. Da die gewählten Minderheitenselbstverwaltungen laut Gesetz die Trägerschaft übernehmen können, und falls das für Bildung und Unterricht zuständige Ministerium diese Übernahme genehmigt und auch die staatliche Finanzierung und Sicherheit vorhanden ist, mussten lediglich einige Monate vergehen und schon wurden in einigen Ortschaften solche Übernahmen geplant. Da landesweite Bildungsinstitutionen seitens der LdU seit einigen Jahren fachlich exzellente und gute Erfahrungen mit der Trägerschaft der Minderheitenselbstverwaltung aufweisen konnten, waren die Weichen schnell gestellt, um dasselbe auch auf örtlicher Basis durchzuführen.

Selbstverständlich müssen die Anregungen von den politischen Gremien der betroffenen Gemeinde oder Stadt hervorgehen. Die örtliche deutsche Nationalitätenselbstverwaltung kann laut Gesetz die Übernahme anregen, der Gemeinde- oder Stadtrat muss das Vorhaben ebenfalls unterstützen. Eben hier haben vielerorts die lokalen Politiker die Übernahme auch deswegen befürwortet, weil in dieser Form doch die örtlichen politischen Vertreter der deutschen Selbstverwaltung, also vor Ort die Entscheidungen treffen werden können. Im Jahre 2015 wurden 14 Kindergärten und Grundschulen in die Trägerschaft von deutschen Selbstverwaltungen übernommen, gewöhnlich in so traditionell „starken“ ungarndeutschen Gemeinden wie in Südungarn Wemend/Véménd, wo der Kindergarten übernommen wurde, oder in Ödenburg die Deutsche Nationalitätenschule. Die LdU hat das Ziel, dieses System von selbst getragenen Minderheiteninstitutionen aus- und aufzubauen, weil ansonsten die oben angeführten negativen Tendenzen immer stärker die gesamte Situation mitprägen würden. Aktuell konnten schon in über 50 Fällen die örtlichen deutschen Selbstverwaltungen Kindergärten und Grundschulen in eigene Trägerschaft übernehmen, da die lokalen Entscheidungsträger das Gefühl hatten, so bleibt die Institution wenigstens unter Aufsicht von einem Gremium, das nicht im fernen Budapest sitzt. Die ersten Erfahrungen widerspiegeln einen vorsichtigen Optimismus und es ist zu hoffen, dass in den nächsten Jahren auch die inhaltliche Arbeit in diesen Bildungsinstitutionen immer mehr der Zielsetzung der Neubelebung der deutschen Sprache und Kultur dienlich sein wird.

Eine wichtige Frage hierbei ist, welches Deutsch - also welche Varietät der deutschen Sprache - im Laufe von diesem Neubelebungsprozess als Zielsprache eingesetzt wird. Interessanterweise werden auch in den Schichten, die noch deutsche Dialektkenntnisse aufweisen können, die Kommunikationsdefizite der Dialekte scharf erfasst 
und bewertet. Die Eltern und Großeltern fordern, dass die Kinder oder Enkelkinder in der Schule die Standardsprache erlernen sollen (vgl. Erb/Knipf 1999: 183). Daraus ergibt sich die Frage, welche Standardvarietät hierbei zu benutzen ist. Da in der Zeit der kommunistischen Diktatur nach einiger Zeit an den germanistischen Lehrstühlen häufiger Lektoren aus der ehemaligen DDR tätig waren, kann behauptet werden, dass bis zur Wende, die Normvorstellungen dieser Personen in der Ausbildung der Deutschlehrer erschienen sind.

Da es eher unrealistisch ist, die einzelnen Dorfdialekte als die Zielsprache der Neubelebung der deutschen Sprache zu bestimmen, aber auch eine nördlich geprägte, eher als eine Wunschvorstellung als sprachliche Realität vorhandene deutsche Standardaussprache nicht als optimale Zielsetzung gelten kann, geben uns Untersuchungen zu den sog. regionalen Standardsprachen Auskunft darüber, welches Deutsch wohl am besten zu unserem Ziel führen könnte (vgl. Berend/Knipf-Komlósi 2006 und Berend 2005 bzw. Földes 2013a und 2013b). Dass die österreichischen Besonderheiten und die in Ungarn traditionell vorhandenen süddeutschen Ausdrücke bei den regionalen Varianten der einzelnen Lexeme (also Topfen neben Quark oder Rauchfang neben Schornstein z.B.) zu berücksichtigen sind, liegt auf der Hand.

Im Allgemeinen wäre es also wichtig, eine süddeutsch geprägte Standardform, eventuell eine bairisch-österreichisch geprägte regionale Standardvarietät als Zielsprache zu benutzen, da ja sowohl in Westungarn als auch im Ungarischen Mittelgebirge die süddeutschen Dialekte dominant sind, allerdings nicht in Südungarn, wo die dialektale Grundlage in Richtung einer hessischen und fuldischen regionalen Sprachform zeigt. In dieser Region bietet der hessische Sprachraum mit seinen alltagssprachlichen Besonderheiten als regionale Standardvarietät einen entsprechenden Hintergrund, um eine mögliche Zielsprache erschließen zu können. Methodisch und sprachlich wäre diese Zielsetzung mit neuen Herausforderungen verbunden, aber meiner Meinung nach würden sich diese Anstrengungen lohnen, um die in Ungarn vorhandenen alten deutschen Sprachtraditionen aufrechtzuerhalten. Dabei könnten die restlichen Dialektkompetenzen der Schüler in dieser Revitalisierung der deutschen Sprache und Kultur mitintegriert werden. Die Kompetenzbestimmung und der Nationale Grundlehrplan (vgl. Müller 2011) brachten zwar in den letzten Jahren eine gewisse Modernisierung der Unterrichts in den bilingualen Schulen der Ungarndeutschen mit sich, der oben angeführten grundsätzlichen Fragestellung bezüglich der zielsprachlichen Varietät und der Integration der dialektalen Restkompetenzen wird allerdings dadurch nicht Rechnung getragen.

\section{ZUSAMMENFASSUNG UND AUSBLICK}

Durch Vertreibung geschwächt und im kommunistischen Ungarn weitestgehend assimiliert kämpft die deutsche Minderheit - wie oben dargestellt - für eine sichere Zukunft und Neubelebung der deutschen Sprache und Kultur. Die diversen Identitätsmuster unter Angehörigen der deutschen Minderheit variieren auf der individuellen Ebene stark. Falls die Revitalisierung der deutschen Sprache und Kultur gelingt, wird dadurch 
die deutsche Identitätskomponente modernisiert und verstärkt. Wenn also eine Neubelebung der deutschen Identität ins Visier genommen wird, können die Ergebnisse der offiziellen Volkszählungen einen entsprechenden Hintergrund in Bezug auf eine gewisse positive Tendenz in der Identität der Angehörigen der deutschen Minderheit zeigen. Anhand der statistischen Daten ergibt sich folgendes Bild, wobei es zu berücksichtigen ist, dass in der offiziellen Statistik infolge der historischen Ereignisse tendenziell niedrigere Angaben zu finden sind. Es ist zu erwähnen, dass die Vertreibung von etwa 200.000 Deutschen in den Jahren 1946-48 auf Grund der Daten der amtlichen (und nicht anonymen) Volkszählung aus dem Jahre 1941 erfolgte. Die Daten der letzten Volkszählungen 2001 und 2011 haben allerdings im Falle der deutschen Minderheit wieder eine steigende Tendenz gezeigt. Schon beim letzten Mal, im Vergleich zur Volkzählung 1990, stieg die Anzahl derer, die im Jahre 2001 als Nationalität Deutsch angegeben haben, von ca. 36.000 auf 62.000, und fast 90.000 Personen haben eine starke Bindung zur Kultur der deutschen Minderheit angegeben. Die Ergebnisse der letzten Volkszählung im Jahre 2011 zeigten in dieser Hinsicht sehr erfreuliche Tatsachen. Das erste Mal konnte bei den Angaben zur Muttersprache eine Trendwende festgehalten werden: Im Jahre 2001 gaben 33.774 Personen Deutsch als ihre Muttersprache an, die Zahl wuchs 2011 auf 38.248. Auch diejenigen, die im familiären oder Bekanntenkreis Deutsch reden, erfuhren in der Statistik ein kräftiges Plus: 2001 waren es 53.000, jetzt sind es 96.000 Personen. Die positivste Entwicklung der neuen Ergebnisse ist zweifelsohne die Verdoppelung der Angaben zur deutschen Nationalität: Der positive Trend wurde nahtlos fortgesetzt und nachdem bereits im Jahre 2001 registrierten kraftvollen Zuwachs, stieg die Zahl derjenigen ungarischen Staatsbürger, die als Nationalität Deutsch angegeben haben im Jahre 2011 auf beachtliche 132.000 Personen.

Des Weiteren ist wichtig zu betonen, dass auch die Ungarndeutschen, die ihre sprachliche Bindung zum Deutschen verloren haben, aber noch eine Restidentität besitzen, einen sehr großen Wert darauf legen, dass ihre Kinder wenigstens in der Schule die deutsche Standardsprache erlernen. Das bedeutet, dass es hier auch darum geht, im Falle einer überintegrierten Minderheit, die sich sprachlich weitgehend assimiliert hat, den Versuch zu unternehmen, Prozess des Sprachwechsels in Richtung Ungarisch zu unterbrechen. Falls dieser Versuch mit Hilfe eines gut ausgebauten zweisprachigen und langfristig auch z. T. einsprachigen Unterrichtswesens nicht gelingt, führt dies zur vollkommenen Assimilation der Deutschen in Ungarn. Ob es gelingen kann, hängt von vielschichtigen Faktoren $a b$. Es gibt Beispiele für eine gelungene Reaktivierung einer fast schon in Vergessenheit geratenen Sprache (z. B. Hebräisch, Katalanisch), aber der Erfolg ist v. a. davon abhängig, ob die staatlichen Institutionen dieser Aufgabe positiv gegenüberstehen und sie unterstützen. Die „Neubelebungsattitüde“ (vgl. Molnár 1998) - also der Wille seitens der Minderheitengruppe zur Belebung der Sprache - ist unter den Deutschen in Ungarn nach meiner Einschätzung vorhanden. Die Angehörigen der Minderheit, die eine Restidentität besitzen, sind wie angedeutet häufig der Meinung, dass wenigstens ihre Kinder und Enkelkinder Deutsch auf einem hohen Niveau beherrschen sollten. Unter den Jugendlichen der Minderheit wirken v. a. die positiven Signale aus der Wirtschaft und der erweiterte europäische Horizont stimulierend. In Ungarn entscheidet sich die Zukunft der deutschen Minderheit im 21. Jahrhundert in den nächsten Jahren und Jahrzehnten. Es ist zu hoffen, 
dass diese Anstrengungen dazu führen werden, den Ungarndeutschen im 21. Jahrhundert eine sichere Zukunftsperspektive zu geben. In den nächsten Jahren wird sich entscheiden, inwiefern die deutsche Minderheit ihr spezifisches Gruppenbewusstsein (wieder)erlangen kann und ob die geschilderten Vorgänge zu einer tatsächlichen Revitalisierung der deutschen Sprache und Kultur in Ungarn führen können.

\section{Literatur}

BEREND, Nina (2005) „Variation ja, aber welche? Zur Frage der Vermittlung von sprachlichen Varianten im Unterricht Deutsch als Fremdsprache.“ In: W. Roggausch (Hrsg.), Germanistentreffen Deutschland - Großbritannien, Irland 30.9.3.10.2004. Bonn: DAAD, 279-296.

BEREND, Nina/Elisabeth KNIPF-KOMLÒSI (2006) „Sprachliche Variation als Herausforderung für den Deutschunterricht in Osteuropa." In: E. Neuland (Hrsg.), Variation im heutigen Deutsch: Perspektiven für den Sprachunterricht. Frankfurt a. Main: Peter Lang, 161-175.

BRENNER, Koloman (1994) „Das Schulwesen der deutschen Volksgruppe in Ungarn. “ In: W. Holzer/U. Pröll (Hrsg.), Mit Sprachen leben. Klagenfurt: Drava Verlag, 135-146.

BRENNER, Koloman (2003) „Sprachliche Situation der deutschen Minderheit in West-Ungarn.“ In: G. Ruda (Hrsg.), Minderheitenschulen - Zweisprachiger Unterricht. Hamburg: Verlag Dr. Kovac, 119-126.

BRENNER, Koloman (2014) „Deutsch(e) in Mittel-Ost-Europa.“ In: Ch. Bergner/H. Zehetmair (Hrsg.), Deutsch als Identitätssprache der deutschen Minderheiten. München: Hanns-Seidel-Stiftung, 71-74.

BRENNER, Koloman (2016) „Neue Tendenzen der Mehrsprachigkeit in Ödenburg und Umgebung.“ In: M. Jesenšek (Hrsg.), Rojena v narečje: akademikinji prof. dr. Zinki Zorko ob 80-letnici. Marburg/Maribor: Filozofska fakulteta, Mednarodna založba Oddelka za slovanske jezike in književnosti, 342--355.

BRENNER, Koloman (2018) Deutsche Minderheit(en) und Institutionen. Budapest: ELTE Germanistisches Institut.

DEMETER ZYZON, Mária (Hrsg.) (1999) Kisebbségek Magyarországon. [Minderheiten in Ungarn.]. Budapest: NEKH.

DOBOS, Balázs (2009) „Hiányosságok és korrekciók a magyarországi kisebbségpolitikában." [Mängel und Korrektionen in der ungarischen Minderheitenpolitik.] Barátság, XVI./3, 6095-6098.

ERB, Maria/Elisabeth KNIPF (1999) „A magyarországi németek körében végzett nyelvismereti felmérés tanulságai.“ [Erkenntnisse der Sprachgebrauchsuntersuchung unter den Ungarndeutschen.] Kisebbségkutatás, 2, 176-187.

FÖLDES, Csaba (2013a): „Deutschunterricht im didaktischen Bezugsraum zwischen Mutter-, Fremd- und Zweitsprache. Fachgeschichtliche und sprachenpolitische Reflexionen anhand des Beispiels Ungarn." In: F. Grucza (Hrsg.), Vielheit und Einheit in der Germanistik weltweit. Frankfurt a. Main (etc.): Peter Lang, 225-233. 
FÖLDES, Csaba (2013b): „Sprachliche Praktiken im Spannungsfeld von Variation und Mehrsprachigkeit: Ein Beitrag zur Empirie.“ In: K. Schneider-Wiejowski/B. Kellermeister-Rehbein/J. Haselhuber, Jakob (Hrsg.), Vielfalt, Variation und Stellung der deutschen Sprache. Berlin/Boston: de Gruyter, 119-142.

FÖLDES, Csaba (2014): „Deutsch im (ost-)mitteleuropäischen Areal. Zwischen kultureller Koexistenz und sprachlicher Symbiose.“ In: M.-V. Lazarescu (Hrsg.), Deutsch als Fremd-und Muttersprache im mitteleuropäischen Raum. Berlin: Wissenschaftlicher Verlag, 87-117.

KNIPF, Elisabeth/Maria ERB (1998): „Sprachgewohnheiten bei den Ungarndeutschen." Beiträge zur Volkskunde der Ungarndeutschen, 15. 138-146.

KNIPF-KOMLÓSI, Elisabeth (2011) Wandel im Wortschatz der Minderheitensprache. Am Beispiel des Deutschen in Ungarn. ZDL, Beihefte, 145. Stuttgart: Franz Steiner Verlag.

MOLNÁR, Helga (1998) „Újratanulható-e az anyanyelv a magyarországi kisebbségi iskolákban?" [Kann die Muttersprache in den Minderheitenschulen Ungarns neu gelernt werden?] Kisebbségkutatás, 3, 321-323.

MÜLLER, Márta (2011) „Über Kompetenzen im DAM-Unterricht der zweisprachigen Minderheitenschulen." In: DUFU - Deutschunterricht für Ungarn 1-2, 154-160.

MÜLLER, Márta (2012) „Formen und Nutzen des ungarndeutschen Minderheitenunterrichts.“ In: G. Kerekes/M. Müller (Hrsg.), Traditionspflege und Erneuerung. Perspektiven der deutschen Nationalität in Ungarn im 21. Jahrhundert. Budapest: Neue-Zeitung-Stiftung, 99-116.

SCHEURINGER, Hermann (2015) „Auf dem Schachbrett der Nationen und Nationalitäten. Die höchst unterschiedlichen Geschichten der und des Deutschen in Mittel-, Ost- und Südosteuropa.“ In: R. Brdar-Szabó/E. Knipf-Komlósi/R. V. Rada (Hrsg.), Zur Rolle und Positionierung des Deutschen in den Ländern Mittelosteuropas. Sprachpolitische Überlegungen. Budapest: Germanistisches Institut, 29-44.

SEEWANN, Gerhard (2012) Geschichte der Deutschen in Ungarn. 1 und 2. Marburg: Herder-Institut.

TAKÁCS, Gerda (2013) „A német nemzetiségi oktatás rendszere napjainkban.” [Das System des deutschen Nationalitätenunterrichts in unseren Tagen.] Neveléstudomány, 2, 76-89.

TÓTH, Ágnes (2015) „A német nemzetiségi oktatás újjászervezése Magyarországon.” [Die Neuorganisierung des deutschen Nationalitätenunterrichts in Ungarn.] Levéltári Szemle, 1, 29-43. 


\section{Zusammenfassung \\ NEUE TENDENZEN IM BILDUNGSSYSTEM \\ DER DEUTSCHEN MINDERHEIT IN UNGARN}

Die deutsche Minderheit in Ungarn gehört zu den relativ großen deutschen Gemeinschaften in Ost-Mittel-Europa, die eine lange historische Entwicklung vorweisen kann. Im heutigen Ungarn gibt es drei größere Siedlungsgebiete, wo Angehörige der deutschen Minderheit in höherer Anzahl leben: Westungarn entlang der österreichischen Grenze, das Ungarische Mittelgebirge (vom Ofner Bergland bis zum Plattensee-Oberland) mit den Zentren Ofen/Buda und Zirc bzw. Südungarn mit dem Zentrum Fünfkirchen/Pécs. Der Aufsatz stellt die neuen Tendenzen im Bildungsbereich und ihre Rolle im Revitalisierungsprozess der deutschen Sprache und Kultur der betroffenen nationalen Minderheit in Ungarn dar. Dies ist auch deswegen eine relevante Fragestellung, weil im Vergleich mit anderen deutschen Minderheiten in Ost-Mittel-Europa, der deutsche Fremdsprachenunterricht und auch der Unterricht von Deutsch als Minderheitensprache eine spezifische Entwicklung vorweisen kann.

Die Landesselbstverwaltung der Ungarndeutschen, als gewähltes Organ der Ungarndeutschen hat das Ziel, ein System von selbst getragenen Minderheiteninstitutionen aus- und aufzubauen. Aktuell konnten schon in über 50 Fällen die örtlichen Deutschen Selbstverwaltungen Kindergärten und Grundschulen in eigene Trägerschaft übernehmen, die ersten Erfahrungen widerspiegeln einen vorsichtigen Optimismus und es ist zu hoffen, dass in den nächsten Jahren auch die inhaltliche Arbeit in diesen Bildungsinstitutionen immer mehr der Zielsetzung der Neubelebung der deutschen Sprache und Kultur dienlich sein wird.

Schlüsselwörter: Deutsche in Ungarn, nationale Minderheiten, Minderheitenunterricht, Deutsch als Minderheitensprache, Ungarn

\section{Abstract \\ NEW TRENDS IN THE EDUCATION SYSTEM OF THE GERMAN MINORITY IN HUNGARY}

The German minority in Hungary, which has a lomg history, is one of the relatively large German minority communities in Eastern-Central Europe. In Hungary today, there are three large settlement areas, where members of the German minority are concentrated: the west of the country along the Austrian border; the Hungarian hills (from the Buda Highlands to the Lake Balaton area) with Buda/Ofen and Zirc as their centres; and the south of Hungary with Pécs/Fünfkirchen as its centre. The paper presents new trends in education and their role in the process of the revitalization of the language and culture of the German national minorities in Hungary. The question is important in view of a specific development the teaching of German as a foreign and as a minority language has had among the German minority in Hungary in comparison with other German minorities in Eastern and Central Europe. 
The provincial self-government of the Hungarian Germans as an elected body of the Hungarian Germans has the aim to establish and develop a system of self-sustaining minority institutions. Currently the local German self-governments are in a position to take charge of over fifty kindergartens and primary schools. The first experiences give grounds for a cautious optimism and, hopefully, in the coming years the activities of these educational institutions will help in the revitalization of the German language and culture.

Keywords: German in Hungary, national minorities, minority teaching, German as a minority language, Hungary

\section{Povzetek \\ NOVE TENDENCE V IZOBRAŽEVALNEM SISTEMU NEMŠKE MANJŠINE NA MADŽARSKEM}

Nemška manjšina na Madžarskem spada med relativno velike nemške manjšinske skupnosti v vzhodni in srednji Evropi, zanjo pa je značilen dolg zgodovinski razvoj. Na Madžarskem obstajajo danes tri večja naselitvena območja, kjer pripadniki nemške manjšine živijo v večjem številu: na zahodnem Madžarskem ob avstrijski meji, v Madžarskem sredogorju (od Budimskega pogorja do zgornjega Blatnega jezera, mestni središči sta Budim/Buda/Ofen in Zirc) ter na južnem Madžarskem (s središčem v mestu Pécs/Fünfkirchen). Prispevek predstavlja nove tendence na področju izobraževanja in njihovo vlogo $\mathrm{v}$ procesu revitalizacije nemškega jezika in kulture omenjenih narodnih manjšin na Madžarskem. Vprašanje je relevantno, saj imata v primerjavi z drugimi nemškimi manjšinami vzhodne in srednje Evrope pouk nemščine kot tujega jezika in tudi pouk nemščine kot manjšinskega jezika specifičen razvoj.

Deželna samouprava madžarskih Nemcev si je zastavila za cilj, da kot izvoljeni organ madžarskih Nemcev osnuje in zgradi lasten sistem manjšinskih institucij. Aktualno stanje kaže, da so krajevne nemške samouprave postale ustanoviteljice vrtcev in osnovnih šol že v več kot 50 primerih. $V$ teh prvih izkušnjah se zrcali previden optimizem in upati je, da bo vsebinsko delovanje teh izobraževalnih institucij prispevalo k uresničitvi cilja oživitve nemškega jezika in kulture.

Ključne besede: Nemci na Madžarskem, narodne manjšine, manjšinski pouk, nemščina kot manjšinski jezik, Madžarska 